\title{
Work-in-Progress Track Chairs' Welcome
}

$\mathrm{Mu} \mathrm{Mu}$, University of Northampton

Elena Fedorovskaya, Rochester Institute of Technology

It is our great pleasure to welcome you to the Work-in-Progress (WiP) track of the 2017 ACM

International Conference on Interactive Experiences for Television and Online Video (TVX 2017). This year's WiP track continues its tradition of previous TVX conferences in soliciting recent viewpoints, new discoveries, and early-stage design and development in disciplines that are in line with TVX's areas of interest. It provides a unique opportunity for exchanging brave new ideas, receiving feedback and fostering collaborations. To further promote the dissemination of new ideas and earlystage project results, several new initiatives are introduced for the WiP track this year. We offer a Project-in-Progress special track, targeting contributions from ongoing major research initiatives including European Commission-funded or other similar-scale projects for cross-project discussions. Additionally, the WiP authors have the opportunity to present their work as a short pitch presentation at the "Madness session" in the main conference program in addition to physical posters at the conference, and importantly, have the papers included in conference adjunct proceedings indexed in ACM Digital Library.

We received a total number of 17 high quality submissions (including 14 on the standard track and 3 on the Project-in-Progress track) from Europe, America and Asia across many topic areas including alternate realities, art, content production, interaction design, immersive media and media studies. Three submissions participated in the Mentoring Program for feedback and guidance from experienced researchers. Based on reviews from Associate Chairs and other expert reviewers in the field, 10 submissions have been accepted. We also selected one winner of the WiP Best Paper Award.

We would like to thank the authors for providing the initial submissions and then working to prepare their camera-ready papers. We are grateful to the TVX 2017 conference Chairs and Committee members for tremendous support and behind the scenes work to prepare this track. We also would like to thank experienced researchers, who participated in the Mentoring Programme for providing invaluable guidance to the authors. We express our deep appreciation for the reviewers, who worked incredibly hard in providing feedback for authors.

We hope that the WiP track will provide you with a valuable opportunity to exchange ideas with other researchers, developers, designers and artists around the world. 